\title{
UTILIZACION DE LA MORFOLOGIA DE LAS LARVAS MEROCERCOIDES PRESENTES EN MOLUSCOS, EN LA DILUCIDACION DE LA TAXONOMIA DE LAS ESPECIES DE RHODOBOTHRIUM (CESTODA: TETRAPHYLLIDEA)*
}

\author{
USE OF THE LARVAL MORPHOLOGY IN THE ELUCIDATION OF THE \\ TAXONOMY OF TETRAPHYLLIDEAN MEROCERCOIDS BELONGING TO \\ THE GENUS RHODOBOTHRIUM PRESENT IN MOLLUSKS
}

\author{
Juan Carvajal \& Alex Mellado \\ Departamento de Recursos Naturales y Medio Ambiente \& Centro de Investigación de Recursos y Ambientes \\ Costeros i mar, Universidad de Los Lagos. Casilla 557, Puerto Montt, Chile. Email: jcarvajal@ulagos.cl \\ * Financiado por la Dirección de Investigación de la Universidad de Los Lagos.
}

\begin{abstract}
RESUMEN
Los moluscos lamelibranquios han sido señalados como hospedadores intermediarios de los parásitos cestodos del orden Tetraphyllidea. Estos platelmintos culminan sus ciclos de vida en los elasmobranquios. En diversas partes del mundo se ha descrito una infinidad de larvas de cestodos del tipo Scolex pleuronectis parasitando molúscos bivalvos. En Chile, Bahamonde \& López (1962) descubren 2 tipos de parásitos cestodos en la macha: un plerocercoide y un merocercoide, habitando respectivamente el intestino y la masa visceral de Mesodesma donacium Lamarck 1818. Mediante cultivos in vitro y disecciones de intestinos de rayas, se ha logrado observar cómo estas larvas se transforman en parásitos adultos (Hamilton \& Byram 1974, Carvajal 1977, Campbell \& Carvajal 1979, Carvajal et al. 1982). Sin embargo, hasta la fecha no se ha podido clarificar cómo se llega a infectar la macha, tampoco se sabe cuáles son los estados de desarrollo previos al estado merocercoide. Se establece que la morfología externa de las larvas merocercoides proporciona una característica importante en la identificación taxonómica de las especies de Rhodobothrium.
\end{abstract}

Palabras claves: Rhodobothrium, Merocercoides, cestodos, lamelibranquios, parásitos,

\section{ABSTRACT}

Lamellibranch mollusks have been reported as intermediate hosts of Tetraphyllidean cestodes. These platyhelminths end their life cycles in the elasmobranch fishes. Larval cestodes belonging to the Scolex pleuronectis type have been found in different parts of the world parasitizing bivalve mollusks. Bahamonde \& López (1962) described 2 types of Tetraphyllidean cestodes in the Chilean clam Mesodesma donacium: a plerocercoid in the intestine and a merocercoid on the visceral mass. By means of the in vitro culture as well as by dissection of the spiral valves of rays, it was possible to observe how these larvi became adult parasites (Hamilton \& Byram 1974; Carvajal 1977, Campbell \& Carvajal 1979, Campbell \& Carvajal 1982). Nevertheless up to now it has not been possible to clarify 1) How Mesodesma donacium became infested and 2) Which are their developmental stages previous to the merocercoid stage. The importance of the external morphology of the merocercoid larvae in the taxonomic identification of the species belonging to the genus Rhodobothrium is established.

KEYWORDS: Rhodobothrium, merocercoid, cestodes, lamellibranches, parasites. 


\section{INTRODUCCION}

En diversas partes del mundo se ha descrito una gran variedad de larvas de cestodos parasitando moluscos bivalvos, las cuales también se han encontrado en invertebrados planctónicos, poliquetos, isópodos, copépodos, crustáceos, otros moluscos y peces (Dollfus 1974, Cake 1976). En Chile, Bahamonde \& López (1962) describen 2 tipos larvarios de cestodos en la macha: un plerocercoide y un merocercoide, habitando el intestino y la masa visceral de Mesodesma donacium Mediante cultivos in vitro y disecciones de intestinos de peje-águilas, rayas que son los hospedadores definitivos de estos cestodos, se ha logrado observar cómo estas larvas se transforman en parásitos adultos (Carvajal 1977, Campbell \& Carvajal 1979, Carvajal et al. 1982). Sin embargo, hasta la fecha no se ha podido esclarecer cómo se llega a infectar la macha, ni cómo son los estados de desarrollo previos al estado merocercoide, así como dilucidar el ciclo completo de ningún parásito cestodo tetrafilídido. El rol que juegan los moluscos bivalvos en el ciclo de vida de estos cestodos, fue establecido por Mudry \& Dailey (1971), Cake (1976) y Euzet (1979), quienes los consideran verdaderos huéspedes intermediarios de los cestodos tetrafilídidos que culminan su ciclo de vida en los elasmobranquios batoídeos hipotremata consumidores de lamelibranquios. Sin embargo hay otros investigadores que consideran hospedadores paraténicos a estos moluscos (Lena Jarecka 2002, comunicación personal). Un obstáculo para la identificación de estas larvas son los cambios que experimenta el escólex al alcanzar el estado adulto, pues se producen transformaciones y pérdidas de estructuras que impiden la identificación taxonómica de estos parásitos (Chambers et al. 2000, Chervi 2002). Este inconveniente se ha logrado revertir utilizando cultivos in vitro y examinando contenidos estomacales frescos de elasmobranquios con el objeto de visualizar los aparatos de fijación de los juveniles, quienes presentan en ciernes las modificaciones de los adultos. Esto ha podido ser ampliamente ilustrado en la literatura (Hamilton \& Byram 1974, Carvajal 1977, Campbell \& Carvajal 1979, Carvajal et al. 1982, Chambers et al. 2000).

En Chile, el peje-águila Myliobatis chilensis (Philippi 1892) (Chondrichthyes: Myliobatidae) es el hospedador definitivo del parásito Rhodobothrium mesodesmatum cuya distribución geográfi- ca va desde Arica hasta Curiñaco, provincia de Valdivia (Pequeño 1975). En tanto la macha Mesodesma donacium (Lamarck 1818) (Mollusca: Lamellibranchia), su hospedador intermediario que alberga el estado merocercoide, se distribuye desde el sur de Perú hasta el rÍo Inio, extremo sur de la isla de Chiloé (Osorio 2002). Un aspecto interesante es que las machas que están bajo el límite inferior de la distribución sur del hospedador de Rodobothrium no presentan el merocercoide (Carvajal 1996).

Campbell \& Carvajal (1979), en una revisión del género Rhodobothrium estudian el estado merocercoide del parásito de la macha identificado como Proboscidosaccus mesodesmatis (Bahamondes \& López 1962) y establecen la ubicación del estado adulto del parásito en la válvula espiral de Myliobatis chilensis y su posición taxonómica como com. nov. Rhodobothrium mesodesmatum (Campbell \& Carvajal 1975).

El objetivo de este trabajo es revisar todos los estados conocidos de merocercoides en moluscos bivalvos que pertenecen al género Rhodobothrium Linton 1889, con el propósito de evaluar si existen características morfológicas que permitan identificar específicamente sus larvas presentes en los lamelibranquios.

\section{MATERIALES Y METODOS}

Se realizó una revisión de toda la literatura mundial referida al género Rhodobothrium presente en las especies hospedadoras de bivalvos (Tabla I). Con ella se confeccionó una comparación de los caracteres morfológicos de fijación de los merocercoides provenientes de las figuras obtenidas a través de cortes histológicos de las especies hospedadoras de bivalvos, de su distribución geográfica y según su tipo de hospedador.

\section{RESULTADOS}

Los estados merocercoides descritos hasta ahora en la literatura son quistes pedunculados que se caracterizan por presentar distintos grados de granulaciones en su región apical ensanchada, al final de la cual se encuentra un orificio de diverso tamaño por donde sale la larva, cuando el quiste es ingerido por el hospedador definitivo apropiado (Fig. 1). 
Gayana 71(1), 2007

TABla I. Especies de larvas merocercoides del género Rhodobothrium y sus huéspedes bivalvos estudiados. Los nombres de los merocercoides se indican abreviados entre paréntesis y se señala su referencia bibliográfica.

TABLE I. Species of larval merocercoids belonging to the genus Rhodobothrium and their bivalve hosts. The merocercoid names are indicated in parenthesis and the references are also shown.

\begin{tabular}{lll}
\hline Merocercoide & Hospedador bivalvo & Referencia \\
\hline Rhodobothrium $\mathrm{sp} .\left(\mathrm{R}_{1}\right)$ & Donax peruvianus (Deshayes, 1875) & Carvajal \& Escalante (1983) \\
Rhodobothrium sp. $\left(\mathrm{R}_{2}\right)$ & Gari solida $($ Gray, 1828) & Oliva (1989) \\
Rhodobothrium lubeti $\left(\mathrm{Rl}_{1}\right)$ & Mactra solida (Linnaeus, 1758) & Gallien (1949); Anthouard (1963) \\
& & y Dollfus (1964) \\
Rhodobothrium lubeti $\left(\mathrm{Rl}_{2}\right)$ & Ostrea edulis (Linnaeus, 1758) & Dollfus (1974) \\
Rhodobothrium mesodesmatum $\left(\mathrm{R}_{3}\right)$ & Mesodesma donacium (Lamark 1818) & Campbell \& Carvajal (1979) \\
\hline
\end{tabular}
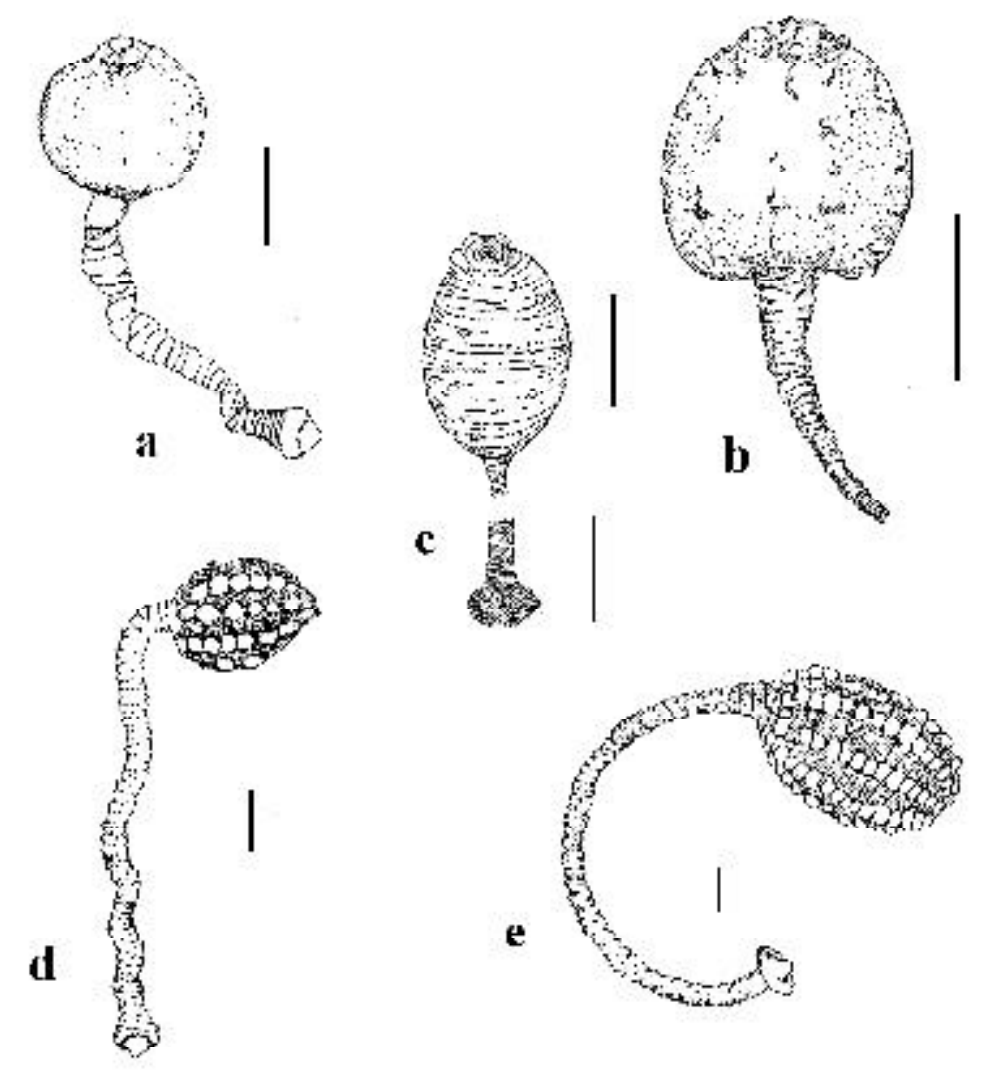

FIGURA 1. Morfología comparada de los estados merocercoides de las especies del género Rhodobothrium descritos en los moluscos lamelibranquios: a) Rhodobothrium sp. de Donax peruvianus, b) Rhodobothrium lubeti de Mactra solida c) Rhodobothrium lubeti de Ostrea edulis, d) Rhodobothrium sp. de Gari solida y e) Rhodobothrium mesodesmatum de Mesodesma donacium. Todas las escalas corresponden a $3 \mathrm{~mm}$.

FIGURE 1. Comparative morphology of the merocercoid stages belonging to the genus Rhodobothrium described in the lamellibranch mollusks: a) Rhodobothrium sp. from Donax peruvianus, b) Rhodobothrium lubeti from Mactra solida c) Rhodobothrium lubeti from Ostrea edulis, d) Rhodobothrium sp. from Gari solida and e) Rhodobothrium mesodesmatum from Mesodesma donacium. All scales correspond to $3 \mathrm{~mm}$. 
Las diferencias externas de los merocercoides van desde la ausencia de tubérculos en la parte quística como es el caso de Rhodobothrium sp. de Donax peruvianus encontrado en Perú (Fig. 1a), pasando por 4 a 5 tubérculos por corrida de gránulos en forma de pezón (como es el caso de Rhodobothrium lubeti de Mactra solida, (Fig. 1b) o circulares y sésiles (sin forma de pezón) como Rhodobothrium lubeti de Ostrea edulis (Fig. 1c), ambos encontrados en Francia. En Chile se han registrado dos morfologías distintas a las descritas más arriba, un merocercoide Rhodobothrium sp., caracterizado por tener 6 a 8 tubérculos por corrida y encontrado en Gari solida en Antofagasta (Fig. 1d) y Rhodobothrium mesodesmatum, merocercoide encontrado en Mesodesma donacium desde Arica a Algarrobo y caracterizado por presentar de 9 a 15 tubérculos por corrida (Fig. 1e).

Diferencias entre las distintas especies de merocercoides de Rhodobothrium, de acuerdo a la localidad, hospedadores intermediarios y definitivos, distribución geográfica, además de algunas características morfológicas, son mostradas en la Tabla II. Las diferencias de tamaño entre los merocercoides encontrados en las distintas especies de bivalvos pueden ser apreciadas en la Fig.1.

\section{DISCUSION}

El gran número de estados larvales de cestodos encontrados en numerosas especies de moluscos en el hemisferio norte, y descritos bajo la denominación de Scolex pleuronectis (Euzet 1959, Goldstein 1967, Williams, 1969), han demostrado ser en su gran mayoría plerocercoides de Acanthobothrium (Hamilton \& Byram 1974, Brooks \& Brothers 1974, Cake 1976, 1979). En Chile los estados plerocercoides encontrados en bivalvos corresponden al cestodo Caulobothrium myliobatidis (Carvajal 1977), presente en la macha (ver Carvajal 1977) y a plerocercoides de Acanthobothrium y Rhinebothrium encontrados en Argopectens purpuratus (Lamark 1819) (ver Oliva et al. $2004 \mathrm{y}$ Oliva \& Sánchez 2005). Se desconoce el número de moluscos parasitados con estas larvas en Chile, así como el impacto económico que ello pueda ocasionar. Por esto que es de vital importancia el estudio descriptivo de los cestodos tanto adultos como larvarios, con el objeto de proporcionar una base en el estudio de sus ciclos evolutivos, especificidad parasitaria, además de la patología que estos parásitos puedan producir en sus hospedadores, sea como adultos o larvas.

En relación al tipo larvario denominado merocercoide, éste fue descrito por Gallien (1949) en la macha Mactra solida de la playa de Saint-Malo, Francia, y debido a que no se pudo asignar a ningún taxón existente en ese entonces, es que se denominó Proboscidosaccus enigmaticus (Gallien 1949). Posteriormente Bahamonde \& López (1962) encuentran en las machas provenientes de Tongoy, Chile, un parásito similar al descrito por Gallien y lo identifican como Proboscidosaccus mesodesmatis, incluyéndolos en el Phylum Platyhelminthes, Orden Tetraphyllidea y Familia Phyllobothriidae. Independientemente dos investigadores franceses: Anthouard (1963) y Dollfus (1964) identifican el taxon al cual pertenece la larva presente en Mactra solida y lo sitúan, al igual que Bahamonde \& López (1962), en el orden Tetraphyllidea y la familia Phyllobothriidae. Dollfus (1974) encuentra a esta misma larva Proboscidossacus enigmaticus en ostras Ostrea edulis de la Bahía de Quiberon, Francia, e indica que el profesor Euzet sugiere que el estado adulto corresponde al cestodo Sphaerobothrium lubeti (Euzet 1959) (= Rhodobothrium lubeti) que se encuentra en el intestino del peje-águila Myliobatis aquila (Lamark 1758). Sin embargo, las dos formas larvales encontradas en Francia presentan características morfológicas y hospedadores intermediarios diferentes, lo que podría indicar que pudieran ser distintas especies.

Posteriormente, Campbell \& Carvajal (1979) revisan la taxonomía de las larvas merocercoides y de sus estados adultos encontrados en los elasmobranquios Myliobatiformes y colocan a Proboscidossacus, Sphaerobothrium e Inermiphyllidium como sinónimos de Rhodobothrium, quedando el parásito de la macha como Rhodobothrium mesodesmatum (Bahamonde \& López 1962) comb. nov. Posteriormente Carvajal et al. (1982) cultivan in vitro merocercoides de $R$. mesodesmatum, y logran obtener estados juveniles y adultos con proglotización y segmentos inmaduros del cestodo con aparato genital esbozado. Carvajal \& Escalante (1983) describen otro merocercoide en la almeja Donax peruvianus, proveniente de las playas de Salaverry, Perú cuyo estado adulto probablemente sea el parásito descrito por Rego et al. 
(1968) como Anthobothrium peruanum (Rego, Vicente \& Herrera 1968) y su hospedador definitivo, Myliobatis peruvianus (Garman 1913). Finalmente, Oliva (1989) encuentra una larva merocercoide de Rhodobothrium en la almeja Gari solida proveniente de una playa de Antofagasta, la cual se diferencia de $R$. mesodesmatum por po- seer un pedúnculo más grueso y un menor número de tubérculos por corrida (ver Tabla II). Estas diferentes características de los merocercoides permite establecer que la morfología externa de estas larvas es una valiosa herramienta de ayuda en la identificación taxonómica de las especies de Rhodobothrium.

Tabla II. Especies del género Rhodobothrium según caracteres morfológicos de sus larvas merocercoides (letras en negrita), especies hospedadoras y origen geográfico (letras sin negrita) descritos en la literatura mundial. Se indican valores en $\mathrm{mm}$ y la información ausente $(*)$ o desconocido (?).

TABLE II. Morphological features between different Rhodobothrium $\left(\mathrm{R}_{1}\right.$ and $\mathrm{R}_{2}=$ Rhodobothrium sp.; $\mathrm{Rl}_{1}$ and $\mathrm{Rl}_{2}=$ Rhodobothrium lubeti $\mathrm{R}_{3}=$ Rhodobothrium mesodesmatum) merocercoid larvae (letters in bold) geographical origins and type of host species (normal letters) described in the literature. Values are in mm., and absent information $(*)$ or unknown (?).

\begin{tabular}{llllll}
\hline \multirow{2}{*}{ Característica } & \multicolumn{5}{c}{ Especie } \\
\cline { 2 - 6 } & $\mathrm{R}_{1}$ & $\mathrm{Rl}_{1}$ & $\mathrm{Rl}_{2}$ & $\mathrm{R}_{2}$ & $\mathrm{R}_{3}$ \\
\hline Número de tubérculos por corrida & $*$ & $4-5$ & $4-5$ & $6-8$ & $9-15$ \\
Abertura apical & 0,9 & 1,99 & 1,2 & $*$ & 1,5 \\
Ventosa del pedúnculo & 1,5 & $*$ & 2,36 & 1,6 & 4,1 \\
Hospedador intermediario & D. peruvianus & M. solida & O. edulis & G. solida & M. donacium \\
Hospedador definitivo & M. peruvianus & M. aquila & M. aquila & $?$ & M. chilensis \\
Localidad geográfica & Perú & Francia & Francia & Chile & Chile \\
\hline
\end{tabular}

\section{DEDICATORIA}

Dedicamos este trabajo al profesor Nibaldo Bahamonde Premio Nacional de Ciencias y a la Profesora María Teresa López (Q.E.P.D.), quienes publicaron el primer trabajo de parasitología de bivalvos marinos en Chile.

\section{BIBLIOGRAFIA}

Anthouard, M. 1963. Contribution a l'étude de Proboscidosaccus enigmaticus Gallien de Mactra solida L. Thesis. Fac. Sc. Nancy. France.

Bahamonde, N. \& M.T. López. 1962. Proboscidosaccus mesodesmatis n. sp. parásito de Mesodesma donacium Lamarck. Investigaciones Zoológicas Chilenas. 8: 43-56.

Brook, D. R. \& E. B. Brothers. 1974. Helminths of three species of goby (Pisces: Gobiidae) from Mision Bay, San Diego. Journal of Parasitology 60: 1062-1063.

Campbell, R. \& J. Carvajal. 1979. Synonymy of the Phyllobotriid genera Rhodobothrium Linton, 1889, Inermiphyllidium Riser, 1955, and Sphaerobothrium Euzet, 1959 (Cestoda:
Tetraphyllidea). Proceedings of the Helminthological Society of Washington 46: 8897.

Carvajal, J. 1977. Description of the adult and larva of Caulobothrium myliobatidis sp. n. (Cestoda: Tetraphyllidea) from Chile. Journal of Parasitology 63: 99-103.

Carvajal, J. 1996. Role of the bivalve molluscs in the life cycle of tetraphyllidean tapeworms. Journal of Medical \& Applied Malacology 8: 20-21.

Carvajal, J., C. Barros \& G. Santander. 1982. In vitro culture of Rhodobothrium mesodesmatum (Cestoda: Tetraphyllidea) parasite of a Chilean clam. Helminthological Society of Washington 49: 226-230.

Carvajal, J. \& H. Escalante. 1983. Nota sobre una larva de cestodo parásito en la almeja peruana Donax peruvianus. Parasitología al Día 7: 89-90.

CAKE, E. 1976. A key to larval cestodes of shallow waters, benthic mollusks of the northern Gulf of Mexico. Proceedings of the Helminthological Society of Washington 43: 160-171.

CAKe, E. 1979. Polypocephalus sp. (Cestoda: Lecanicephalidea): a description of tentaculoplerocercoids from bay scallops of the northeastern Gulf of Mexico. Proceeding of the Helminthological Society of Washington 46: 165170. 
Chambers, C. B., T.H. Cribb \& M. Jones. 2000. Tetraphyllidean metacestodes of teleosts of the Great Barrier Reef, and the use of in vitro cultivation to identify them. Folia Parasitologica 47: $285-292$

CHERvi, L. 2002. The terminology of larval cestodes or metacestodes. Systematic Parasitology 52: 1-33.

Pequeño, G. 1975. Nuevo registro de Myliobatis chilensis Philippi (Elasmobranchii: Myliobatidae). Boletín de la Sociedad de Biología de Concepción. 49: 157-160.

Dollfus, R. 1964. Enumération des cestodes du plancton et des invertébrés marins ( $6^{\circ}$ contribution). Annales Parasitologie Humaine \& Comparée 39: 329-379.

Dollfus, R. 1974. Enumération des cestodes du plancton et des invertébrés marins. Avec un appendice sur le genre Oncomegas R. Ph. Dollfus, 1929. Annales Parasitologie Humaine \& Comparée 49: 381-410.

EuzET, L. 1959. Recherches sur les cestodes Tetraphyllides des Sélaciens des Cotes de France. These, Montpellier.

EuZET, L. 1979. Role et place des mollusques daus le cycle evolutif des cestodes. Haliotis 8: 115-120.

Falavigna-Morais, D., L. M achado \& G. Pavanelli. 2003. Protocephalian larvae (Cestoda) in naturally infected cyclopoid copepods of the upper Paraná river flooplain, Brazil. Memórias do Instituto Oswaldo Cruz 98: 69-72.

Gallien, L. 1949. Proboscidosaccus enigmaticus nov. Gen nov.sp. parasite de Mactra solida L. (Note preliminaire). Bulletin Societé Zoologique de France 74: 322-326.

Goldstein, R. J. 1967. The genus Acanthobothrium van
Beneden, 1849 (Cestoda: Tetraphyllidea). Journal of Parasitology 53: 455-483.

Hamilton, K. \& J. Byram. 1974. Tapeworm development: the effects of urea on a larval tetraphyllidean. Journal of Parasitology 60: 20-28.

Mudry, D. \& M. Dailey. 1971. Postembryonic development of certain tetraphyllidean and trypanorhynchan Cestodes with a possible alternative life cycle for the order Trypanorhynchan. Canadian Journal of Zoology 49: 1249-1253.

Oliva, M. 1989. Gari solida (Gray,1828), nuevo huésped para un plerocerco del género Rhodobothrium (Cestoda:Tetraphyllidea). Parasitología al Día 13: 46-47.

Oliva, M., L. Castillo \& M. Sánchez. 2004. Metazoan parasites in wild and farmed population of the scallops, Argopoecten purpuratus (Lamarck, 1819) from northern Chile. Acta Parasitologica 49: $153-155$

Oliva, M. \& M. SÁnchez. 2005. Metazoan parasites and commensals of the northern Chilean scallop Argopecten purpuratus (Lamarck) as a tool for stock identification. Fisheries Research 71: 71-77.

Osorio, C. 2002. Moluscos marinos en Chile, especies de importancia económica. Imprenta Salesianos S. A. $211 \mathrm{p}$.

Rego, A., J. Vicente \& N. Herrera. 1968. Sobre dois novos parasitos de peixe da costa do Peru (Cestoda: Tetraphyllidea). Memórias do Instituto Oswaldo Cruz 66: 145-149.

Williams, H. 1969. The genus Acanthobothrium van Beneden, 1849 (Cestoda: Tetraphyllidea). Nytt Magazine Zoologie 17: 1-56. 